\title{
The effect of density stratification on the amplitude profile of transversal coronal loop oscillations
}

\author{
R. Erdélyi and G. Verth \\ Solar Physics and upper-Atmosphere Research Group (SPARG), University of Sheffield, Hicks Building, Hounsfield Road, \\ Sheffield S3 7RH, UK \\ e-mail: [Robertus;G.Verth]@sheffield.ac.uk
}

Received 25 May 2006 / Accepted 28 September 2006

\section{ABSTRACT}

\begin{abstract}
Context. Measuring how the density of the plasma in the Sun's corona varies along fine structures such as coronal loops using emission measure is very difficult as the exact value of the line of sight filling factor and the correct amount of background subtraction are unknown.

Aims. To investigate if magnetohydrodynamic (MHD) wave theory can be used to complement existing observational methods to obtain diagnostic information about the density structure of coronal loops by measuring the amplitude profile of the fundamental standing mode of the fast kink wave.

Methods. Analytical and numerical approaches are used to show how the amplitude profile of the fundamental fast kink mode changes by varying the amount of density stratification in a coronal loop.

Results. Increasing the amount of density stratification in a coronal loop will increase the difference in amplitude profile from one of constant longitudinal density. For a semi-circular TRACE $171 \AA$ Aemperature loop of length $200 \mathrm{Mm}$ and density scale height, $H=50 \mathrm{Mm}$, the maximum change in amplitude profile is of the order of $50 \mathrm{~km}$. It is also found that the amplitudes of these oscillations are effectively negligible at altitudes lower than the transition region.

Conclusions. The effect of density stratification on the amplitude profile of the fundamental standing kink mode of a TRACE $171 \AA$ temperature loop may be very subtle. Unfortunately, the observational signatures of this effect are likely to be well below the resolution of the best currently available extreme-ultraviolet (EUV) imagers. Only loops with homogeneous magnetic fields have been addressed here, but inhomogeneous magnetic fields may well dominate over the effect of density stratification on the amplitude profile.
\end{abstract}

Key words. Sun: corona - Sun: magnetic fields - Sun: oscillations - Sun: fundamental parameters

\section{Introduction}

High resolution EUV images by the Transition Region And Coronal Explorer (TRACE) have shown many observations of post-flare transversal coronal loop oscillations (see e.g., Aschwanden et al. 1999a, 2002; Nakariakov et al. 1999; Verwichte et al. 2004). Using existing MHD theory by Edwin \& Roberts (1983) describing waves in a magnetic cylinder, Aschwanden et al. (1999a) and Nakariakov et al. (1999) interpreted these transversal loop oscillations as the fundamental standing mode of the fast kink wave. Using these observations, attempts were made at coronal seismology (as suggested initially by Roberts et al. 1984) with the purpose of providing diagnostic information about magnetic field strength along loops. The procedure involved measuring the period of oscillation, loop length and estimating plasma density from emission measure.

A simplification in the theory used so far was that the plasma density and magnetic field strength remained constant along loops. In fact, density decreases dramatically from the photosphere to the corona, e.g., Vernazza et al. (1981) estimated that for a bright active region the number density of electons in the photosphere was $\approx 10^{14}-10^{15} \mathrm{~cm}^{-3}$ and only $\approx 10^{9} \mathrm{~cm}^{-3}$ in the lower corona. Although variation in magnetic field strength along loops cannot be measured directly, it is highly likely that field strength decreases substantially from the photosphere to corona. Assuming a dipole potential field model, the dependence of the magnetic field strength, $B$ on vertical height $h$ above the photosphere ( $h=0$ is the photospheric height) can be given by

$B(z) \approx B_{\text {foot }}\left(1+\frac{h}{h_{\mathrm{d}}}\right)^{-3}$,

where $B_{\text {foot }}$ is the photospheric field strength and $h_{\mathrm{d}}$ is the dipole depth (see e.g., Aschwanden 2004). Using the Solar and Heliospheric Observatory/Extreme-ultraviolet Imaging Telescope (SoHO/EIT), $171 \AA$ images of thirty active region loops that were cospatial with $\mathrm{SoHO} /$ Michelson-Doppler Imager (MDI) magnetograms, Aschwanden et al. (1999b) estimated that $B_{\text {foot }} \approx 20-200 \mathrm{G}$ and $B \lesssim 10 \mathrm{G}$ for $h \gtrsim 100 \mathrm{Mm}$.

Currently, MHD wave theory is being developed to model more realistic loop equilibria, e.g., magnetic cylinders with curvature (Van Doorsselaere et al. 2004), twisted magnetic fields (Bennett et al. 1998; Erdélyi \& Fedun 2006), non-circular crosssections (Ruderman 2003) and density stratification (Díaz et al. 2002; Andries et al. 2005b; Dymova \& Ruderman 2005). The ground work to describe waves in more complex magnetically structured flux tubes has also been addressed, e.g. by Erdélyi \& Carter (2006). In this paper we will implement a method which allows the density to vary arbitrarily along a loop. We will investigate how density stratification affects the spatial displacement of the commonly observed fundamental fast kink mode of loops compared with those of constant longitudinal density and if these effects could be measured by current or future solar missions. 
This is a qualitative shift from a previous approach by Andries et al. (2005a) where attention was paid to data in the frequency domain of transversal loop oscillations.

\section{Theoretical approach}

There are many observational difficulties in determining the density structure of the corona. What would appear to be the most straightforward way of measuring plasma density is by emission measure (where image intensity is proportional to plasma density squared, see e.g., Aschwanden 2004), but this technique is actually plagued by unknown parameters such as the line of sight filling factor (e.g. Cargill \& Klimchuk 1997; Jain \& Yashiro 2002). Also, observers have argued about whether coronal loops are isothermal or non-isothermal along their structure on the basis of interpreting filter ratios (Reale \& Peres 2000) or varying background subtraction (Schmelz et al. 2005).

The theory of MHD waves in magnetic cylinders may be able to complement this work by using observations of coronal loop oscillations to determine how the important parameter of plasma density changes along a loop. Andries et al. (2005a) suggested that by measuring the periods of the fundamental mode and first harmonic of a loop, information can be acquired about the longitudinal density stratification of that loop. Using TRACE only two possible observations have been made of first overtones (Verwichte et al. 2004) but unfortunately the time cadence of the data was too poor to determine the periods to the accuracy required by their model.

Observers have previously used the EUV imager on TRACE to measure the amplitude of fundamental kink mode oscillations in coronal loops (see e.g., Aschwanden et al. 1999a, 2002; Nakariakov et al. 1999; Verwichte et al. 2004). It is with this in mind, that we shall focus on how the amplitude profile of the fundamental kink mode in a coronal loop is affected by changing the density stratification.

The radial velocity component (which is directly proportional to the amplitude) of the fundamental mode for a magnetic cylinder with varying longitudinal density has been calculated before. This was carried out, e.g., by Diaz et al. (2002) for a prominence type density profile and Andries et al. (2005b) for two coronal loop type density profiles. These papers both calculated the standing mode solutions using numerical approaches. The main difference in our present study is that we use analytical approaches whenever possible. We also want to carry out a comprehensive comparative study into how the amplitude profile of the fundamental mode changes by varying the density stratification. Diaz et al. (2002) did not consider this aspect and the results presented by Andries et al. (2005b) did not fully illustrate this very interesting phenomenon.

The magnetic cylinder model we use was suggested by Dymova \& Ruderman (2005). The plasma is considered to be ideal and cold (plasma $\beta=0$ ) which are reasonably valid assumptions for the corona. The full MHD equations can then be simplified to the form,

$$
\begin{aligned}
& \frac{\partial \rho}{\partial t}+\nabla \cdot(\rho \boldsymbol{v})=0, \\
& \rho \frac{\mathrm{D} \boldsymbol{v}}{\mathrm{D} t}=\frac{1}{\mu}(\nabla \times \boldsymbol{B}) \times \boldsymbol{B}, \\
& \frac{\partial \boldsymbol{B}}{\partial t}=\nabla \times(\boldsymbol{v} \times \boldsymbol{B}), \\
& \nabla \cdot \boldsymbol{B}=0,
\end{aligned}
$$

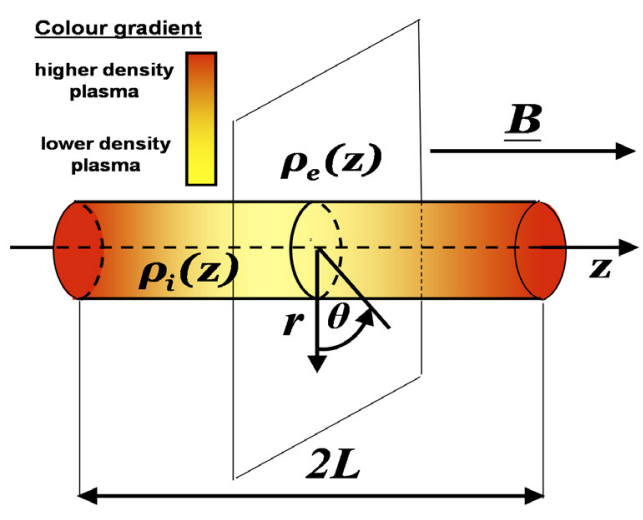

Fig. 1. The equilibrium configuration of a magnetic cylinder with longitudinally varying density.

where $\boldsymbol{B}$ is the magnetic field vector, $\boldsymbol{v}$ is the plasma velocity vector, $\rho$ is the plasma density, $\mu$ is the magnetic permeability and $\mathrm{D} / \mathrm{D} t$ is the material derivative for time variations following the motion (defined by $\mathrm{D} / \mathrm{D} t \equiv \partial / \partial t+\boldsymbol{v} \cdot \nabla$ ).

The model was originally developed to describe the oscillations of prominence fibrils but is general enough to apply to coronal loops since it allows for an arbitrary equilibrium density along the waveguide. Using cylindrical co-ordinates $(r, \phi, z)$, a coronal loop in its equilibrium state is modelled by a straight cylindrical magnetic flux tube of total length $2 L$ and radius $R$ (see Fig. 1). The plasma densities both outside $\left(\rho_{\mathrm{e}}\right)$ and inside $\left(\rho_{\mathrm{i}}\right)$ the tube are assumed to depend only on the longitudinal $z$ co-ordinate. $\boldsymbol{B}$ is directed along the $z$ axis and is constant in magnitude everywhere.

By observation (see e.g. Aschwanden et al. 2002) it is also assumed that $R \ll L$ (the thin flux tube approximation) and that the loop has footpoints fixed somewhere in the lower solar atmosphere where the plasma is of higher density, i.e., $\boldsymbol{v}=\mathbf{0}$ at $z= \pm L$. With these valid assumptions we can linearise Eqs. (2) to (5) (for details see Dymova \& Ruderman 2005) and derive a simple second order ordinary differential equation which describes standing fast kink modes (see e.g., Roberts et al. 1984) of the loop in terms of the maximum radial velocity perturbation, $v_{r}(z)$ at the tube boundary $r=R$, shown by

$$
\frac{\mathrm{d}^{2} v_{r}}{\mathrm{~d} z^{2}}+\frac{\omega^{2}}{c_{k}^{2}(z)} v_{r}=0
$$

with boundary conditions

$v_{r}=0 \quad$ at $\quad z= \pm L$

where $\omega$ is the angular frequency and

$c_{\mathrm{k}}^{2}(z)=\frac{2 B^{2}}{\mu\left(\rho_{\mathrm{i}}(z)+\rho_{\mathrm{e}}(z)\right)}$.

The loop's fixed footpoints described by condition (7) mean that $\omega^{2}$ is an eigenvalue of a Sturm-Liouville problem defined by Eq. (6) and boundary conditions (7). If $\rho_{\mathrm{i}}(z)$ and $\rho_{\mathrm{e}}(z)$ are constant then $c_{\mathrm{k}}$ is known as the kink speed (the characteristic phase speed in the thin tube, long wavelength limit). Here we still call $c_{\mathrm{k}}$ the kink speed even when there is density stratification. In our present study we assume that external density $\rho_{\mathrm{e}}(z)$ is directly proportional to internal density $\rho_{\mathrm{i}}(z)$, such that

$\rho_{\mathrm{e}}(z)=\epsilon \rho_{\mathrm{i}}(z) \quad$ where $\quad \epsilon \in(0,1)$. 
The model used here does not actually require this particular constraint, but it does simplify the mathematical analysis considerably.

Since we actually want to solve for the amplitude of the standing fast kink modes instead of $v_{r}(z)$, we shall introduce $\psi(z)$, the maximum amplitude of the transversal oscillation at position $z$. In standing modes all of the loop oscillates in phase, giving the simple relationship $v_{r}(z)=\omega \psi(z)$. For a SturmLiouville eigenvalue problem, the eigenvalues $\omega^{2}$ form an infinite sequence, and consequently there is also an infinite sequence of eigenfunctions, $\psi(z)$. The eigenfunction associated with each eigenvalue is unique up to a constant scaling factor.

At this point it is appropriate to introduce the subscript notation $n$, where $n=1,2,3,4, \ldots$ This denotes the specific eigenvalue, $\omega_{n}^{2}$ and associated eigenfunction, $\psi_{n}(z)$. When describing standing waves, $n$ is often referred to as the mode number. The analytical approach employed in this present study requires that we solve for odd and even integer modes separately. It is therefore necessary to rewrite (6) and (7) in terms of the specific mode number $n$ we are solving for, i.e.,

$\frac{\mathrm{d}^{2} \psi_{n}}{\mathrm{~d} z^{2}}+\left(\alpha \omega_{n}\right)^{2} \rho_{\mathrm{i}}(z) \psi_{n}=0$

and

$\psi_{n}=0$ at $z= \pm L$

where

$\alpha=\frac{1}{B} \sqrt{\frac{\mu(\epsilon+1)}{2}}$.

Using stereoscopic analysis, Aschwanden et al. (1999b) found that for 30 loops studied in EUV, most fitted closely to an exponentially stratified density model. The loop footpoints were assumed to be about $2.5 \mathrm{Mm}$ above the photosphere. If a coronal loop is semi-circular with an exponentially stratified plasma, then the form of $\rho_{\mathrm{i}}(z)$ would be

$\rho_{\mathrm{i}}(z)=\rho_{\mathrm{f}} \exp \left[-\frac{2 L}{\pi H} \cos \left(\frac{\pi z}{2 L}\right)\right]$,

where $\rho_{\mathrm{f}}$ is the footpoint density of the loop and $H$ is the density scale height. This is equivalent to the form of $\rho_{\mathrm{i}}(z)$ used by Andries et al. (2005a) to study the effect of density stratification on loop oscillations in the frequency domain. Unfortunately, we know of no analytical solution for Eq. (10) when $\rho_{\mathrm{i}}(z)$ is described by (13). We shall solve (10) and (11) with the density profile given by Eq. (13) numerically in Sect. 6 but useful insight into the behaviour of the solution can be gained by using various approximations to (13). It is with this in mind that we have chosen three separate types of approximations to (13) which increase in refinement and mathematical difficulty as we progress from Sects. 3 through to 5 .

\section{Step function density profile}

For our most basic approximation to density profile (13) we shall use step functions. This will most simply illustrate how varying the footpoint and apex densities can change the amplitude profile of a standing kink mode when Eq. (10) is solved with condition (11). Figure 2a shows a comparison between the type of step density function used against the more realistic density profile (13).
Taking the density discontinuity boundaries to be at $z= \pm d$, the density along the loop is defined by

$\rho_{\mathrm{i}}(z)= \begin{cases}\rho_{\mathrm{a}}, & z \in[-d, d] \\ \rho_{\mathrm{f}}, & z \in[-L,-d) \cup(d, L],\end{cases}$

where $\rho_{\mathrm{f}}$ is the footpoint density and $\rho_{\mathrm{a}}$ is the apex density and both are constants. Density profile (14) gives contact discontinuities at $z= \pm d$, therefore the following boundary conditions have to be satisfied (see e.g., Landau \& Lifshitz 1956; Cowling 1960),

$[\boldsymbol{v}]=0, \quad[\boldsymbol{B}]=0$,

where the square brackets indicate the jump of a quantity at $z=$ $\pm d$. This implies that $\psi_{n}(z)$ and its derivative must be continuous at $z= \pm d$ (see Dymova \& Ruderman 2005).

We are primarily interested in the fundamental mode solutions. This is an odd integer mode $(n=1)$, so we shall only be looking at the odd integer mode solutions. A similar mathematical approach can also be used to obtain all the even integer mode solutions. As well as condition (11), the symmetry of density profile (14) about $z=0$, means that all odd integer modes must have

$\psi_{n}(0)=A_{n}$,

$\frac{\mathrm{d} \psi_{n}}{\mathrm{~d} z}(0)=0$,

where $A_{n}$ is the arbitrary maximum amplitude at the loop apex. Solving (10) locally in each region of $z$ and matching the solutions together at the density discontinuity so that $\psi_{n}(z)$ and its derivative are continuous (see e.g., Zwillinger 1997) gives the solution for the amplitude of all odd integer standing modes as,

$\psi_{n}(z)= \begin{cases}A_{n} \cos \left(\alpha \omega_{n} \sqrt{\rho_{\mathrm{a}}} z\right), & z \in[-d, d] \\ B_{n} \cos \left(\alpha \omega_{n} \sqrt{\rho_{\mathrm{f}}} z\right) & \\ +C_{n} \sin \left(\alpha \omega_{n} \sqrt{\rho_{\mathrm{f}}}|z|\right), & z \in[-L,-d) \cup(d, L],\end{cases}$

where

$$
\begin{aligned}
B_{n}= & \frac{A_{n}}{\sqrt{\rho_{\mathrm{f}}}}\left\{\sqrt{\rho_{\mathrm{f}}} \cos \left(\alpha \omega_{n} \sqrt{\rho_{\mathrm{f}}} d\right) \cos \left(\alpha \omega_{n} \sqrt{\rho_{\mathrm{a}}} d\right)\right. \\
& \left.+\sqrt{\rho_{\mathrm{a}}} \sin \left(\alpha \omega_{n} \sqrt{\rho_{\mathrm{f}}} d\right) \sin \left(\alpha \omega_{n} \sqrt{\rho_{\mathrm{a}}} d\right)\right\},
\end{aligned}
$$

and

$$
\begin{aligned}
C_{n}= & \frac{A_{n}}{\sqrt{\rho_{\mathrm{f}}}}\left\{\sqrt{\rho_{\mathrm{f}}} \sin \left(\alpha \omega_{n} \sqrt{\rho_{\mathrm{f}}} d\right) \cos \left(\alpha \omega_{n} \sqrt{\rho_{\mathrm{a}}} d\right)\right. \\
& \left.-\sqrt{\rho_{\mathrm{a}}} \cos \left(\alpha \omega_{n} \sqrt{\rho_{\mathrm{f}}} d\right) \sin \left(\alpha \omega_{n} \sqrt{\rho_{\mathrm{a}}} d\right)\right\},
\end{aligned}
$$

only if the following equation is satisfied (because of the boundary condition [11]),

$$
\begin{aligned}
& \tan \left(\alpha \omega_{n} \sqrt{\rho_{\mathrm{f}}} L\right)= \\
& \quad-\frac{\sqrt{\rho_{\mathrm{f}}}+\sqrt{\rho_{\mathrm{a}}} \tan \left(\alpha \omega_{n} \sqrt{\rho_{\mathrm{a}}} d\right) \tan \left(\alpha \omega_{n} \sqrt{\rho_{\mathrm{f}}} d\right)}{\sqrt{\rho_{\mathrm{f}}} \tan \left(\alpha \omega_{n} \sqrt{\rho_{\mathrm{f}}} d\right)-\sqrt{\rho_{\mathrm{a}}} \tan \left(\alpha \omega_{n} \sqrt{\rho_{\mathrm{a}}} d\right)} .
\end{aligned}
$$

Obviously Eq. (21) is not valid if $\rho_{\mathrm{a}}=\rho_{\mathrm{f}}$. In the case of constant density along the loop $\left(\rho_{i}(z)=\rho_{\mathrm{c}}\right.$, say), solution (18) just simplifies to

$\psi_{n}(z)=A_{n} \cos \left(\alpha \omega_{n} \sqrt{\rho_{\mathrm{c}}} z\right), \quad z \in[-L, L]$, 

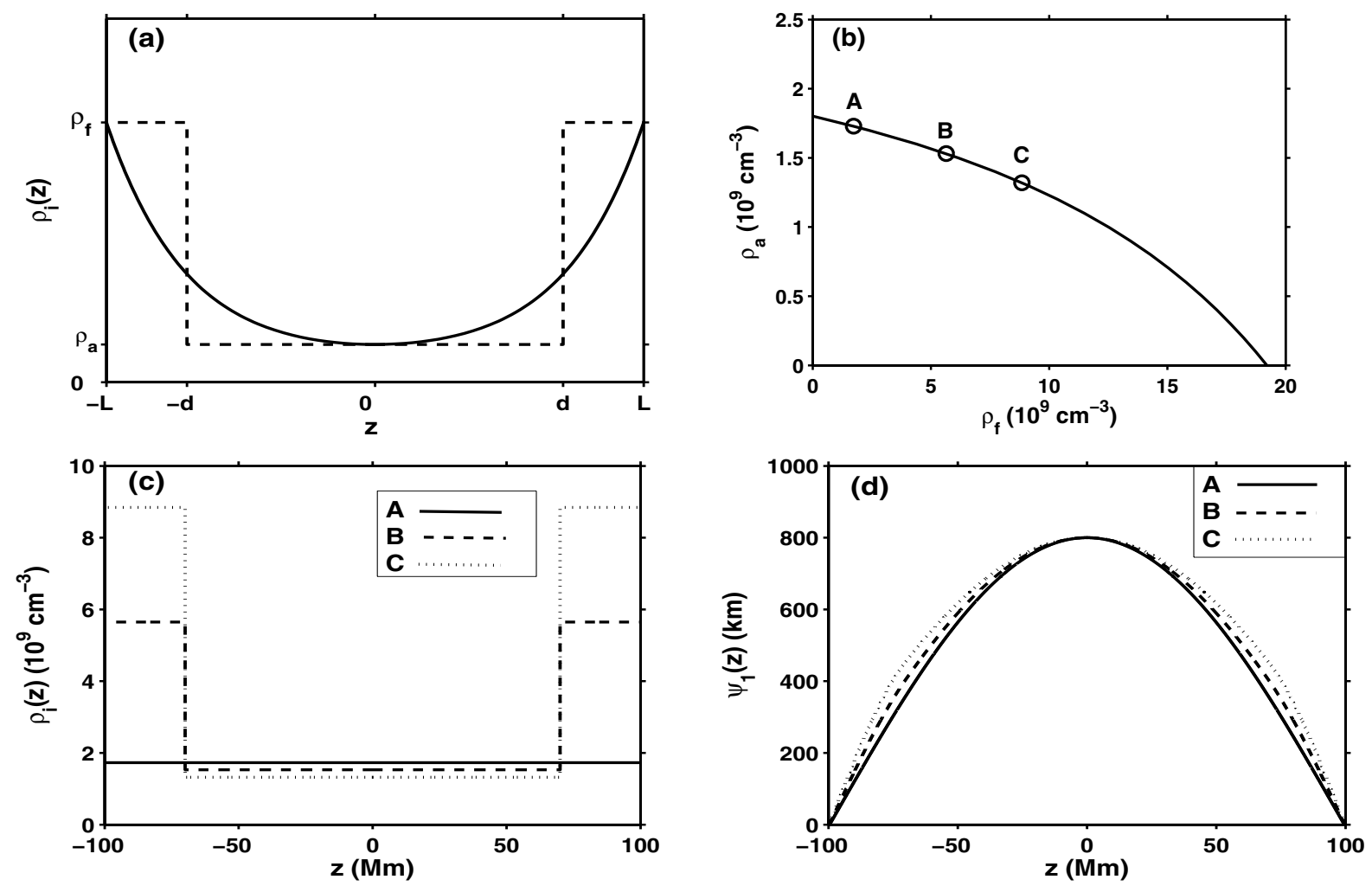

Fig. 2. a) The step function approximation (dashed line) given by Eq. (14) and the exponentially stratified density profile (solid line) given by Eq. (13). b) The allowed density pairs (given in units of elecron number density $\left(10^{9} \mathrm{~cm}^{-3}\right)$ ) for the fundamental standing mode where $\rho_{\mathrm{a}}$ and $\rho_{\mathrm{f}}$ are apex and footpoint densities. The fixed parameters are $L=100 \mathrm{Mm}, d=70 \mathrm{Mm}, B=10 \mathrm{G}, P_{1}=5 \min$ and $\epsilon=0.1$. Pairs A, B and C are chosen to represent $H=\infty, H \approx 50 \mathrm{Mm}$ and $H \approx 33 \mathrm{Mm}$. c) The density profiles of A, B and C. d) The amplitude profiles of A, B and C, where for all, the apex maximum amplitude for the fundamental kink mode (at $z=0$ ) is normalised to $A_{1}=800 \mathrm{~km}$.

where

$\rho_{\mathrm{c}}=\left(\frac{n \pi}{2 \alpha \omega_{n} L}\right)^{2}$.

For the more general case of $\rho_{\mathrm{a}} \neq \rho_{\mathrm{f}}$, we shall look at all possible density stratifications of the fundamental mode $(n=1)$ for fixed $\alpha, d, L$ and $\omega_{1}$ (in this study we shall assume that $\omega_{1}$ is an observed parameter and therefore always fixed). Since the fundamental mode must have $\psi_{1}(z)$ non-zero everywhere on the domain except at $z= \pm L$, it is easy to see from (18) that $\rho_{\mathrm{a}}$ and $\rho_{\mathrm{f}}$ must be within the following ranges,

$0 \leq \rho_{\mathrm{a}}<\left(\frac{\pi}{2 \alpha \omega_{1} d}\right)^{2}$

and

$0<\rho_{\mathrm{f}} \leq\left(\frac{\pi}{2 \alpha \omega_{1}(L-d)}\right)^{2}$.

In fact, the upper limit of $\rho_{\mathrm{f}}$ is exactly as stated in (25) but the upper limit for $\rho_{\mathrm{a}}$ has to be calculated by taking the limit of (21) as $\rho_{\mathrm{f}} \rightarrow 0$, which gives the transcendental equation,

$\tan \left(\alpha \omega_{1} \sqrt{\rho_{\mathrm{a}}} d\right)=\frac{1}{\alpha \omega_{1}(L-d) \sqrt{\rho_{\mathrm{a}}}}$.

The required upper limit for $\rho_{\mathrm{a}}$ is the first positive root of Eq. (26) (higher positive roots give nodes on the domain $z \in$ $[-d, d]$, and therefore do not give fundamental mode solutions).

For each value of $\rho_{\mathrm{f}}$ in the range defined by (25), there is a unique value of $\rho_{\mathrm{a}}$ in the range given by (24) that solves
Eq. (21). These density pairs will each give a unique fundamental kink mode amplitude profile, $\psi_{1}(z)$ when the values of $\rho_{\mathrm{f}}$ and $\rho_{\mathrm{a}}$ are specified in (18). The range of allowed density pairs (given in units of electron number density, $10^{9} \mathrm{~cm}^{-3}$ ) is illustrated in Fig. 2b for fixed values of $d, L, \alpha$ and $\omega_{1}$. The chosen fixed parameter values for the fundamental mode period, $P_{1}$ (where $\left.P_{1}=2 \pi / \omega_{1}\right)$ and loop half length, $L$ are typical of those values observed by TRACE in $171 \AA$ (plasma at $\approx 1 \mathrm{MK}$ ), i.e., $P_{1}=5 \mathrm{~min}$ and $L=100 \mathrm{Mm}$ (see Aschwanden et al. 2002). The other fixed parameter values are chosen to be $d=70 \mathrm{Mm}$ (to approximate the exponentially stratified density profile [13]), $B=10 \mathrm{G}$, a plausible average coronal magnetic field strength (see Aschwanden et al. 1999b) and $\epsilon=0.1$ (see Golub \& Pasachoff 1997).

Using SoHO/EIT, stereoscopic analysis in EUV of coronal loops with plasma at temperatures of between 1-1.5 MK (similar to the TRACE $171 \AA$ passband), Aschwanden et al. (1999b) estimated the average density scale height, $H$ to be $55 \pm 10 \mathrm{Mm}$. In Fig. 2b, three specific density pairs $\mathrm{A}, \mathrm{B}$ and $\mathrm{C}$ are plotted to represent $H=\infty, H \approx 50 \mathrm{Mm}$ and $H \approx 33 \mathrm{Mm}$. These are therefore loops of constant density, typical stratification and more extreme stratification. The density and amplitude profiles for $\mathrm{A}, \mathrm{B}$ an $\mathrm{C}$ are shown in Figs. $2 \mathrm{c}$ and $2 \mathrm{~d}$. In all cases, the value for $A_{1}$, the maximum amplitude at the apex of the loop (at $z=0$ ) is normalised to be $800 \mathrm{~km}$, a typical observed value (see Aschwanden et al. 2002).

It can be noted from Fig. $2 \mathrm{~b}$, that as $\rho_{\mathrm{f}} \rightarrow 0$ there are density pairs where $\rho_{\mathrm{a}}>\rho_{\mathrm{f}}$. This is unphysical for coronal loops, but may be relevant for the kink oscillations of prominences. Most importantly, the difference between the amplitude profiles 

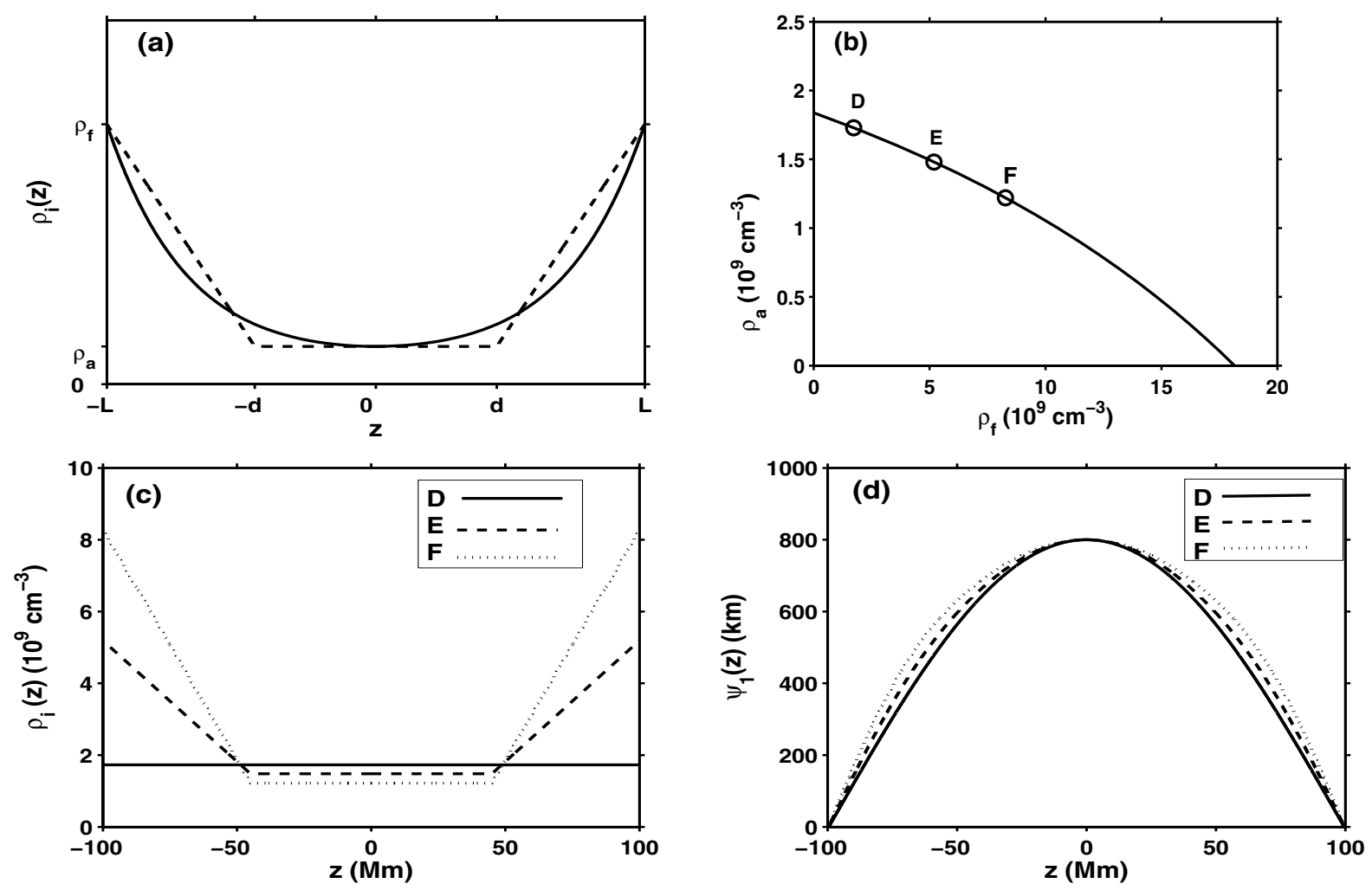

Fig. 3. a) The piecewise linear approximation (dashed line) given by Eq. (27) and the exponentially stratified density profile (solid line) given by Eq. (13). b) The allowed density pairs for the fundamental standing mode where $\rho_{\mathrm{a}}$ and $\rho_{\mathrm{f}}$ are apex and footpoint densities. The fixed parameters are $L=100 \mathrm{Mm}, d=45 \mathrm{Mm}, B=10 \mathrm{G}, P_{1}=5 \mathrm{~min}$ and $\epsilon=0.1$. Pairs D, E and $\mathrm{F}$ are chosen to represent $H=\infty, H \approx 50 \mathrm{Mm}$ and $H \approx 33 \mathrm{Mm}$. c) The density profiles of D, E and F. d) The amplitude profiles of D, E and F, where for all, the apex maximum amplitude for the fundamental kink mode (at $z=0$ ) is normalised to $A_{1}=800 \mathrm{~km}$.

is subtle as can be seen clearly in Fig. 2d. Even for the more extreme stratification case $\mathrm{C}(H \approx 33 \mathrm{Mm})$, the loop apex is still only a few scale heights above the photosphere. For an average TRACE $171 \AA$ A temperature loop in the corona one might expect $H \approx 50 \mathrm{Mm}$ (profile B). The step density profile (14) approximation to that of a semi-circular TRACE $171 \AA$ temperature loop with $H \approx 50 \mathrm{Mm}$ (profile $\mathrm{B}$ ) has a maximum difference in amplitude profile from the one of constant density (profile A) of approximately $30 \mathrm{~km}$ and this occurs at about $30 \mathrm{Mm}$ from the footpoints.

To find $\psi_{n}(z)$ for the higher odd integer modes $(n=$ $3,5,7, \ldots$ ) for a particular density pair shown in Fig. $2 b$, we use the chosen values of $\rho_{\mathrm{a}}$ and $\rho_{\mathrm{f}}$ in (21) to find the relevant $\omega_{n}$ (with $\alpha, d$ and $L$ still fixed at the same values). Since $\omega_{n}^{2}$ is the eigenvalue of the Sturm-Liouville boundary value problem (10) and (11), for $n=1,2,3,4, \ldots$, the sequence $\left\{\omega_{n}^{2}\right\}$ is monotonically increasing (see e.g. Coddington \& Levinson 1955). Now treating $\omega_{n}$ as the free parameter, for a fixed $\rho_{\mathrm{f}}$ and $\rho_{\mathrm{a}}$ pair the lowest positively valued root of (21) is the frequency of the fundamental mode, $\omega_{1}$ (which is already known) and the higher valued positive roots of (21) form the monotonically increasing sequence $\left\{\omega_{n}\right\}$, where $n=3,5,7, \ldots$ Hence, for any density pair $\rho_{\mathrm{f}}$ and $\rho_{\mathrm{a}}$, all the odd integer mode values of $\omega_{n}$ for $n \geq 3$ can be calculated and then used in (18) to find the corresponding amplitude profiles, $\psi_{n}(z)$.

\section{Linear density profile}

Increasing the complexity of the density profile slightly, a linear function is chosen in the regions $z \in[-L,-d) \cup(d, L]$, as shown in Fig. 3a. The density function is now piecewise continuous, given by

$\rho_{\mathrm{i}}(z)= \begin{cases}\rho_{\mathrm{a}}, & z \in[-d, d] \\ \frac{\left(\rho_{\mathrm{f}}-\rho_{\mathrm{a}}\right)|z|+\left(L \rho_{\mathrm{a}}-d \rho_{\mathrm{f}}\right)}{L-d}, & z \in[-L,-d) \cup(d, L] .\end{cases}$

As before, if $\rho_{\mathrm{a}}=\rho_{\mathrm{f}}$ then the solution of (10) is just described by (22) and (23), but if $\rho_{\mathrm{a}} \neq \rho_{\mathrm{f}}$ then the solution in the regions $z \in$ $[-L,-d) \cup(d, L]$ is now described in terms of Airy functions Ai and $\mathrm{Bi}$. So the amplitude function for all odd integer modes is now of the form

$\psi_{n}(z)= \begin{cases}A_{n} \cos \left(\alpha \omega_{n} \sqrt{\rho_{\mathrm{a}}} z\right), & z \in[-d, d] \\ B_{n} \operatorname{Ai}\left(-\left(\frac{\alpha \omega_{n}}{a}\right)^{\frac{2}{3}}(a|z|+b)\right) & \\ +C_{n} \operatorname{Bi}\left(-\left(\frac{\alpha \omega_{n}}{a}\right)^{\frac{2}{3}}(a|z|+b)\right), & z \in[-L,-d) \cup(d, L],\end{cases}$

such that

$a=\frac{\rho_{\mathrm{f}}-\rho_{\mathrm{a}}}{L-d}$,

$b=\frac{L \rho_{\mathrm{a}}-d \rho_{\mathrm{f}}}{L-d}$

$B_{n}=A_{n} \pi\left\{\mathrm{Bi}^{\prime}\left(-\xi_{\mathrm{d}}\right) \cos \left(\eta_{\mathrm{d}}\right)\right.$

$$
\left.-\left(\frac{\alpha \omega_{n}}{a}\right)^{\frac{1}{3}} \sqrt{\rho_{\mathrm{a}}} \operatorname{Bi}\left(-\xi_{\mathrm{d}}\right) \sin \left(\eta_{d}\right)\right\}
$$

$C_{n}=-A_{n} \pi\left\{\mathrm{Ai}^{\prime}\left(-\xi_{\mathrm{d}}\right) \cos \left(\eta_{\mathrm{d}}\right)\right.$

$$
\left.-\left(\frac{\alpha \omega_{n}}{a}\right)^{\frac{1}{3}} \sqrt{\rho_{\mathrm{a}}} \operatorname{Ai}\left(-\xi_{\mathrm{d}}\right) \sin \left(\eta_{\mathrm{d}}\right)\right\},
$$


and

$\eta_{\mathrm{d}}=\alpha \omega_{n} \sqrt{\rho_{\mathrm{a}}} d$,

$\xi_{\mathrm{d}}=\left(\frac{\alpha \omega_{n}}{a}\right)^{\frac{2}{3}}(a d+b)$.

The relationship that for all values of $x$, the Wronskian, $W\{\operatorname{Ai}(x), \operatorname{Bi}(x)\}=\pi^{-1}$ (Abramowitz \& Stegun 1965), was used to simplify the expressions for the coefficients (31) and (32). Boundary conditions (11) mean that (28) is only a solution for odd integer modes if

$$
\frac{\operatorname{Ai}\left(-\xi_{L}\right)}{\operatorname{Bi}\left(-\xi_{L}\right)}=\frac{\operatorname{Ai}^{\prime}\left(-\xi_{\mathrm{d}}\right)-\left(\frac{\alpha \omega_{n}}{a}\right)^{\frac{1}{3}} \sqrt{\rho_{\mathrm{a}}} \operatorname{Ai}\left(-\xi_{\mathrm{d}}\right) \tan \left(\eta_{\mathrm{d}}\right)}{\mathrm{Bi}^{\prime}\left(-\xi_{\mathrm{d}}\right)-\left(\frac{\alpha \omega_{n}}{a}\right)^{\frac{1}{3}} \sqrt{\rho_{\mathrm{a}}} \mathrm{Bi}\left(-\xi_{\mathrm{d}}\right) \tan \left(\eta_{\mathrm{d}}\right)},
$$

where,

$\xi_{L}=\left(\frac{\alpha \omega_{n}}{a}\right)^{\frac{2}{3}}(a L+b)$.

Again, for the fundamental mode $(n=1)$ we shall look at all the possible density stratifications for fixed values of $\alpha, d, L$ and $\omega_{1}$, using Eq. (35) to find the upper limits for $\rho_{\mathrm{a}}$ and $\rho_{\mathrm{f}}$. When $\rho_{\mathrm{a}}=0$, the upper limit for $\rho_{\mathrm{f}}$ is the first positive root of

$\frac{\operatorname{Ai}\left(-\left(\chi_{1} \sqrt{\rho_{\mathrm{f}}}\right)^{\frac{2}{3}}\right)}{\operatorname{Bi}\left(-\left(\chi_{1} \sqrt{\rho_{\mathrm{f}}}\right)^{\frac{2}{3}}\right)}=-\frac{1}{\sqrt{3}}$,

where

$\chi_{1}=\alpha \omega_{1}(L-d)$.

The relationship that for all values of $x, \operatorname{Ai}^{\prime}(x) / \operatorname{Bi}^{\prime}(x)=-1 / \sqrt{3}$ (Abramowitz \& Stegun 1965) was used in deriving Eq. (37). When $\rho_{\mathrm{f}}=0$, the upper limit for $\rho_{\mathrm{a}}$ is the first positive root of

$$
\begin{aligned}
& -\left[\chi_{1} \sqrt{\rho_{\mathrm{a}}}\right]^{\frac{1}{3}} \tan \left(\alpha \omega_{1} \sqrt{\rho_{\mathrm{a}}} d\right)= \\
& \frac{\sqrt{3} \mathrm{Ai}^{\prime}\left(-\left[\chi_{1} \sqrt{\rho_{\mathrm{a}}}\right]^{\frac{2}{3}}\right)-\mathrm{Bi}^{\prime}\left(-\left[\chi_{1} \sqrt{\rho_{\mathrm{a}}}\right]^{\frac{2}{3}}\right)}{\sqrt{3} \mathrm{Ai}\left(-\left[\chi_{1} \sqrt{\rho_{\mathrm{a}}}\right]^{\frac{2}{3}}\right)-\operatorname{Bi}\left(-\left[\chi_{1} \sqrt{\rho_{\mathrm{a}}}\right]^{\frac{2}{3}}\right)} .
\end{aligned}
$$

The relationship that for all values of $x, \operatorname{Ai}(x) / \operatorname{Bi}(x)=1 / \sqrt{3}$ (Abramowitz \& Stegun 1965) was used in deriving Eq. (39).

The fixed equilibrium loop parameters are the same as used in Sect. 3 for $\alpha, L$ and $\omega_{1}$ but now $d=45 \mathrm{Mm}$ to approximate the exponentially stratified profile (13) more closely. From Eqs. (37) and (39) the range limits for the allowed density pairs $\rho_{\mathrm{f}}$ and $\rho_{\mathrm{a}}$ are found. Within these ranges Eq. (35) is solved and the resultant density pairs are plotted in Fig. $3 \mathrm{~b}$. The linear density profile (27) approximation to that of a semi-circular TRACE $171 \AA$ temperature loop with $H \approx 50 \mathrm{Mm}$ (profile E) gives the maximum difference in amplitude from one of constant density (profile D) as approximately $35 \mathrm{~km}$, occurring at about $35 \mathrm{Mm}$ from the loop footpoints.

It can be seen that the linear approximation density pairs curve in Fig. $3 \mathrm{~b}$ closely matches that of the step approximation shown in Fig. 2b, as do the fundamental mode amplitude profiles in Figs. $3 \mathrm{~d}$ and $2 \mathrm{~d}$. The small amplitude differences between linear and step cases $\mathrm{E}$ and $\mathrm{B}$ (loops with $H \approx 50 \mathrm{Mm}$ ) are shown in Fig. 4. The maximum differences are only about $\pm 7 \mathrm{~km}$ and

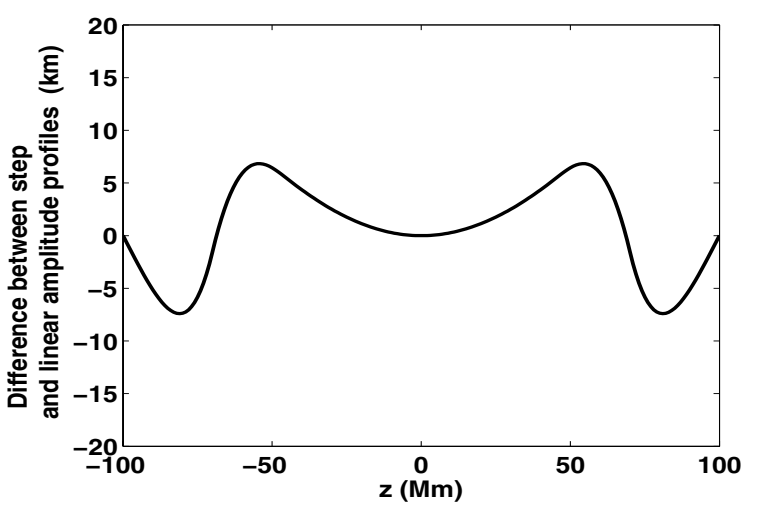

Fig. 4. Difference between the step and linear amplitude profiles (14) and (27) for $L=100 \mathrm{Mm}$ and $H \approx 50 \mathrm{Mm}$, i.e., profile $\mathrm{E}$ - profile B.

occur at $\pm 55 \mathrm{Mm}$ and $\pm 82 \mathrm{Mm}$. To attempt the inverse problem to distinguish between the linear or step cases $\mathrm{E}$ and B, i.e., measuring an amplitude profile $\mathrm{E}$ or $\mathrm{B}$ and then calculating the correct density profile using Eq. (10) with condition (11), one would obviously have to measure the amplitude profile to the required accuracy, i.e., to less than $7 \mathrm{~km}$.

The exponentially stratified density model fitted by Aschwanden et al. (1999b) to EUV observations was valid for loop footpoints assumed to be $2.5 \mathrm{Mm}$ above the photosphere. The linear density profile (27) can actually be used as a reasonable approximation of the solar atmosphere from the photosphere through to the transition region and corona to model what the amplitude profile of the standing kink mode would look like if the footpoints were fixed at the photosphere. It has been estimated, e.g. by Vernazza et al. (1981), that from the photosphere to the transition region the density decreases by 5-6 orders of magnitude. For this model $\rho_{\mathrm{f}}$, the photospheric electron number density at $z= \pm L$ is taken to be $10^{15} \mathrm{~cm}^{-3}$ and this decreases linearly to the transition region at $z= \pm d$ (2 Mm from the photosphere) where $\rho_{\mathrm{a}}$, the coronal density is $10^{9} \mathrm{~cm}^{-3}$. The coronal density is assumed to be constant since here we are only interested in how the very large density gradient between the photosphere and the transition region affects the fundamental mode solution of (10) with boundary conditions (11). This linear density approximation to the density gradient between the photosphere and the corona is shown in Fig. 5a.

A shorter length loop is chosen with $L=50 \mathrm{Mm}$ (with $A_{1}=800 \mathrm{~km}$ ) to better illustrate the behaviour of the amplitude solution in the $2 \mathrm{Mm}$ region between the photosphere and the transition region. It is found that there is only one solution that resembles TRACE observations of fundamental kink modes in the coronal segment of the loop, i.e., an amplitude profile that decreases in size from the loop apex towards the footpoints (see Fig. 5b). In the loop segments between the transition region and the photosphere, this solution actually has many nodes and a vastly reduced amplitude (as shown in Fig. 5c). The very steep density gradient at the transition region is acting like a barrier, forcing the kink mode to have a node at this point. In each $2 \mathrm{Mm}$ loop footpoint segment between the photosphere and the transition region there are 15 nodes and the maximum amplitude decreases to less than $5 \mathrm{~km}$. Strictly speaking, the solution is not a pure fundamental mode but as the amplitude of the oscillation becomes effectively negligible at the transition region, the loop is behaving as if the footpoints are actually fixed in the transition region. Often in observations the fixed footpoints of the fundamental kink mode are assumed to be in the much denser photosphere. The study here shows that this is probably a less 
accurate assumption and that they could be at least $2 \mathrm{Mm}$ higher in the solar atmosphere.

\section{Hyperbolic cosine density profile}

For a final analytical insight, to model a continuous and smoothly curved density profile the hyperbolic cosine function is used as follows,

$\rho_{\mathrm{i}}(z)=\rho_{\mathrm{a}} \cosh \left[\cosh ^{-1}\left(\frac{\rho_{\mathrm{f}}}{\rho_{\mathrm{a}}}\right) \frac{z}{L}\right]$.

Qualitatively, density profile (40) models (13) very well as can be seen from Fig. 6a. Now Eq. (10) can be solved using Mathieu functions, $\mathrm{C}$ and $\mathrm{S}$. The odd integer modes are given by

$\psi_{n}(z)=A_{n} \mathrm{C}\left(0,2 \rho_{\mathrm{a}}\left[\frac{\alpha \omega_{n} L}{\cosh ^{-1}\left(\frac{\rho_{\mathrm{f}}}{\rho_{\mathrm{a}}}\right)}\right]^{2}, \frac{i}{2} \cosh ^{-1}\left(\frac{\rho_{\mathrm{f}}}{\rho_{\mathrm{a}}}\right) \frac{z}{L}\right)$

as long as the boundary condition (11) is satisfied, i.e.,

$\mathrm{C}\left(0,2 \rho_{\mathrm{a}}\left[\frac{\alpha \omega_{n} L}{\cosh ^{-1}\left(\frac{\rho_{\mathrm{f}}}{\rho_{\mathrm{a}}}\right)}\right]^{2}, \frac{i}{2} \cosh ^{-1}\left(\frac{\rho_{\mathrm{f}}}{\rho_{\mathrm{a}}}\right)\right)=0$.

Again for the fundamental mode $(n=1)$, by fixing $\alpha, \omega_{1}$ and $L$, we can easily find the upper limit of $\rho_{\mathrm{a}}$ by making $\rho_{\mathrm{f}}=0$ in (42). The upper limit for $\rho_{\mathrm{a}}$ is the first positive root of

$\mathrm{C}\left(0,-2 \rho_{\mathrm{a}}\left(\frac{2 \alpha \omega_{1} L}{\pi}\right)^{2},-\frac{\pi}{4}\right)=0$.

By Eq. (42), there is now no upper limit for $\rho_{\mathrm{f}}$ since $\rho_{\mathrm{f}} \rightarrow \infty$ as $\rho_{\mathrm{a}} \rightarrow 0$. This is shown clearly in Fig. 6b, where we have solved Eq. (42) for fixed parameters $\alpha, L$ and $\omega_{1}$ (with the same values as in the previous sections). The upper limit for $\rho_{\mathrm{f}}$ in the previous case studies of the step and linear density profiles in Sects. 3 and 4 was due to the parameter $d$ being fixed. The plots of allowed density pairs in Figs. $2 b$ and $3 b$ would have been similar to Fig. 6b if we had allowed $d$ to vary such that $d \rightarrow L$ as $\rho_{\mathrm{f}} \rightarrow \infty$ and $\rho_{\mathrm{a}} \rightarrow 0$.

The hyperbolic cosine density profile approximation (40) to that of a semi-circular TRACE $171 \AA$ temperature loop with $H \approx$ $50 \mathrm{Mm}$ (profile $\mathrm{H}$ ) has a maximum amplitude difference with the loop of constant density (profile $\mathrm{G}$ ) of about $42 \mathrm{~km}$ at a distance $38 \mathrm{Mm}$ from the loop footpoints (as shown in Fig. 6d).

\section{Example of an exponentially stratified density profile}

Finally, using the most realistic exponentially stratified density profile (13), Eq. (10) with boundary conditions (11) is solved for the fundamental mode numerically using the shooting method (see e.g., Zwillinger 1997). The same fixed values for $\alpha, L$ and $\omega_{1}$ are used. Using the symmetry about $z=0$, the shooting method is started at $z=0$ for the fundamental mode with fixed values $\psi_{1}=800 \mathrm{~km}$ and $\mathrm{d} \psi_{1} / \mathrm{d} z=0$ (see [16] and [17]). For any chosen value of $H \in(-\infty, 0) \cup(0, \infty), \rho_{\mathrm{f}}$ can be adjusted to an arbitrary accuracy to obtain the fundamental mode solution, i.e., $\psi_{1}( \pm L)=0$ and $\psi_{1}(z) \neq 0$ for $z \in(-L, L)$.

The resulting numerical calculations are shown in Fig. 7. Obvious qualitative similarities can be seen between the plots for allowed density pairs, density and amplitude profiles for the hyperbolic cosine density approximation loop shown in Figs. 6b-d
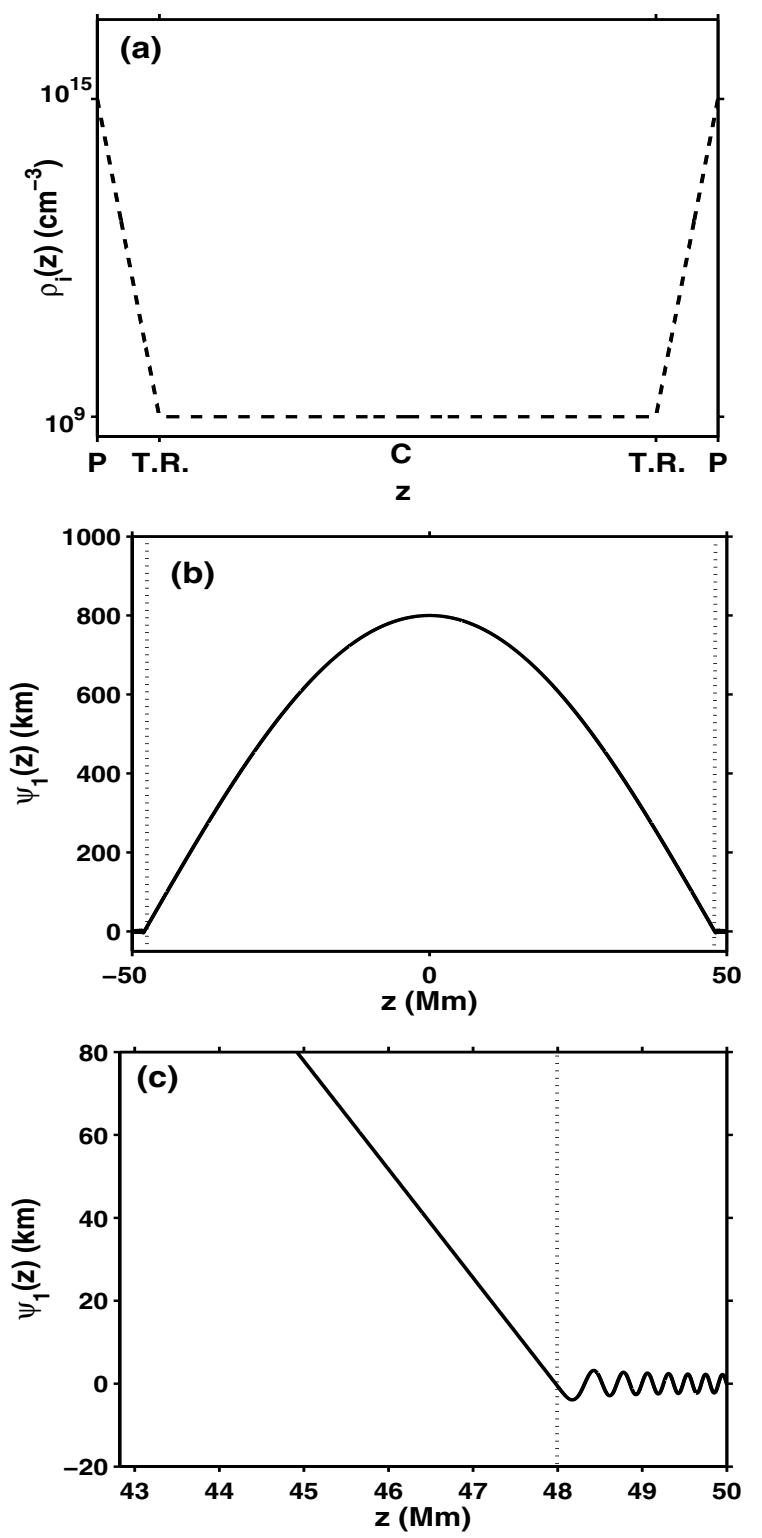

Fig. 5. a) Linear approximation of the density profile from the photosphere $(\mathrm{P})$ through the transition region (T.R.) to the corona (region C), where the distance between the P and T.R. is $2 \mathrm{Mm}$. b) The quasi-fundamental standing mode amplitude solution for a loop with $L=50 \mathrm{Mm}$ and $A_{1}=800 \mathrm{~km}$. The position of the T.R. is shown by the vertical dotted line. c) A blow-up of the amplitude profile at the right hand footpoint shown in (b).

and those of the exponentially stratified one shown in Figs. 7a-c. A semi-circular exponentially stratified TRACE $171 \AA$ A temperature loop with $H=50 \mathrm{Mm}$ (profile $\mathrm{K}$ ) has a maximum amplitude difference with the loop of constant density (profile L) of about $46 \mathrm{~km}$ at a distance $38 \mathrm{Mm}$ from the loop footpoints.

\section{Summary and conclusions}

A fundamental question of great interest is: could the simple second order differential Eq. (10) with boundary conditions (11) be used to determine how the density profile changes along a coronal loop by observing the spatial dependence of the amplitude? To answer this question fully we would have to investigate the relative contributions of all other physical effects that could vary the amplitude profile which have not been addressed here. It is 

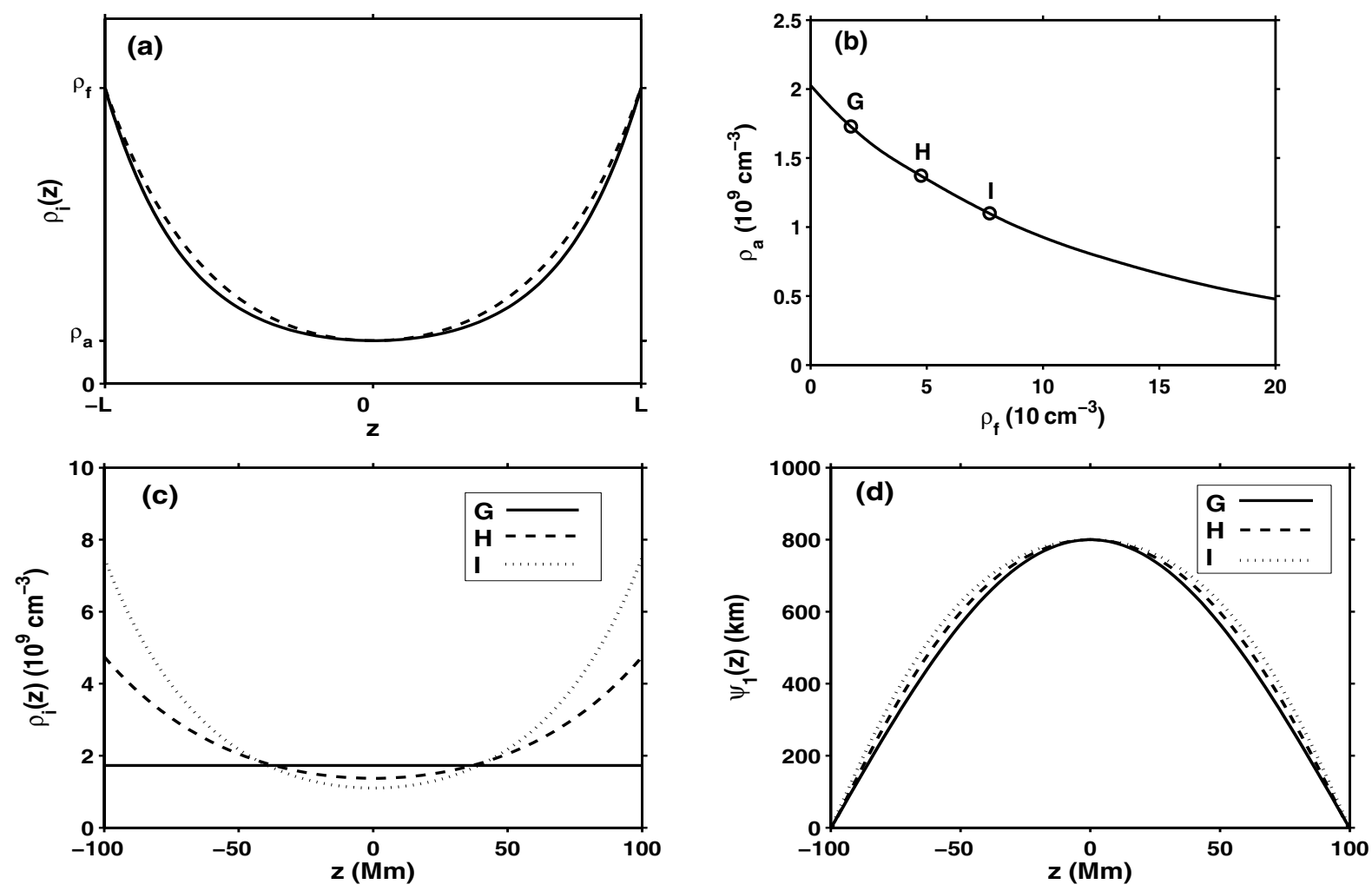

Fig. 6. a) The hyperbolic cosine function approximation (dashed line) given by Eq. (40) and the exponentially stratified density profile (solid line) given by Eq. (13). b) The allowed density pairs for the fundamental standing mode where $\rho_{\mathrm{a}}$ and $\rho_{\mathrm{f}}$ are apex and footpoint densities. The fixed parameters are $L=100 \mathrm{Mm}, B=10 \mathrm{G}, P_{1}=5 \mathrm{~min}$ and $\epsilon=0.1$. Pairs $\mathrm{G}, \mathrm{H}$ and I are chosen to represent $H=\infty, H \approx 50 \mathrm{Mm}$ and $H \approx 33 \mathrm{Mm}$. c) The density profiles of G, H and I. d) The amplitude profiles of G, H and I, where for all, the apex maximum amplitude for the fundamental kink mode (at $z=0$ ) is normalised to $A_{1}=800 \mathrm{~km}$.

also possible that the magnetic field decreasing in strength from the loop footpoints to the apex could be the dominant factor since the kink speed, $c_{\mathrm{k}}$ is proportional to $B$, the magnitude of the magnetic field. In the model presented here, the magnetic field was kept constant to make the problem more tractable. To our current knowledge, there is not yet a model that describes the standing fast kink mode of a magnetic cylinder that has a magnetic field changing in strength along its axis, except a work in progress by ourselves.

The exact magnetic structure along coronal loops is still very much a mystery as there has not yet been a technique developed which can provide a direct measurement. The indirect observational evidence so far has been rather puzzling. A study of TRACE loops (Watko \& Klimchuk 2000) has shown that the cross-sectional width remains relatively constant with increasing height above the photosphere. The flux tube interpretation suggests that magnetic field is therefore almost constant along loops but this contradicts potential and force-free field extrapolations from $\mathrm{SoHO} / \mathrm{MDI}$ data where the field lines always diverge with height. Klimchuk et al. (2000) suggested that by twisting a loop this could reduce the amount of width expansion with height. They performed a force-free extrapolation with a twisted loop embedded in a magnetic dipole and found that although the twist did reduce the expansion of the loop they could still not match the observed constant thickness. It would therefore be useful to develop theoretical models which could predict how different magnetic field structures in loops would affect the frequency and spatial properties of loop oscillations. These models could then be tested against observations and help further advance the field of coronal seismology.
It was shown in the previous sections that density stratification in a TRACE $171 \AA$ temperature coronal loop with a constant magnetic field is likely to give only a subtle change to the amplitude profile of fundamental fast kink mode oscillations compared to one of constant longitudinal density (see Table 1 for comparisons). The effect is greatest when a loop's apex is very much higher than its density scale height. This agrees with the result of Andries et al. (2005a) concerning changes within frequency domain. A typical semi-circular TRACE $171 \AA$ temperature loop with exponential density stratification $(L=100 \mathrm{Mm}$ and $H=50 \mathrm{Mm}$ ) will only differ in amplitude from one of constant density by a maximum of $46 \mathrm{~km}$. TRACE, our current best resolution EUV imager has a pixel size equivalent to about $357 \mathrm{~km}$ on the surface of the Sun (and therefore has a resolution of nearly $714 \mathrm{~km}$ ), so this difference is far too small to be measured at present.

There are also many practical problems for observers in measuring spatial dependence of amplitude along a loop. Currently, it is very difficult to use 2D images from TRACE to infer the true 3D geometry of a loop. Aschwanden et al. (2002) have tried circular fits using heliospheric co-ordinates with limited success. The planned Solar TErrestrial RElations Observatory (STEREO) mission may be of help in this regard as it will view the Sun using two imagers simultaneously from two different locations giving quasi-3D data to work from (but unfortunately its EUV Imaging Telescope (EUVI) has about three times less resolution than TRACE).

Furthermore, since TRACE can only use one EUV passband at a time to view the coronal plasma, only part of the loop is usually clearly visible. This is because loop temperature can vary in 
Table 1. Comparison of results from Sects. 3-6.

\begin{tabular}{ccc}
\hline \hline $\begin{array}{c}\text { Density profile approximation } \\
\text { of semi-circular stratified loop } \\
(L=100 \mathrm{Mm} \text { and } H \approx 50 \mathrm{Mm})\end{array}$ & $\begin{array}{c}\text { Maximum amplitude difference }(\mathrm{km}) \\
\text { to loop with constant density }\end{array}$ & $\begin{array}{c}\text { Distance from footpoints (Mm) } \\
\text { where maximum difference occurs }\end{array}$ \\
\hline step & 30 & 30 \\
linear & 35 & 35 \\
hyperbolic cosine & 42 & 38 \\
exponential & 46 & 38 \\
\hline
\end{tabular}
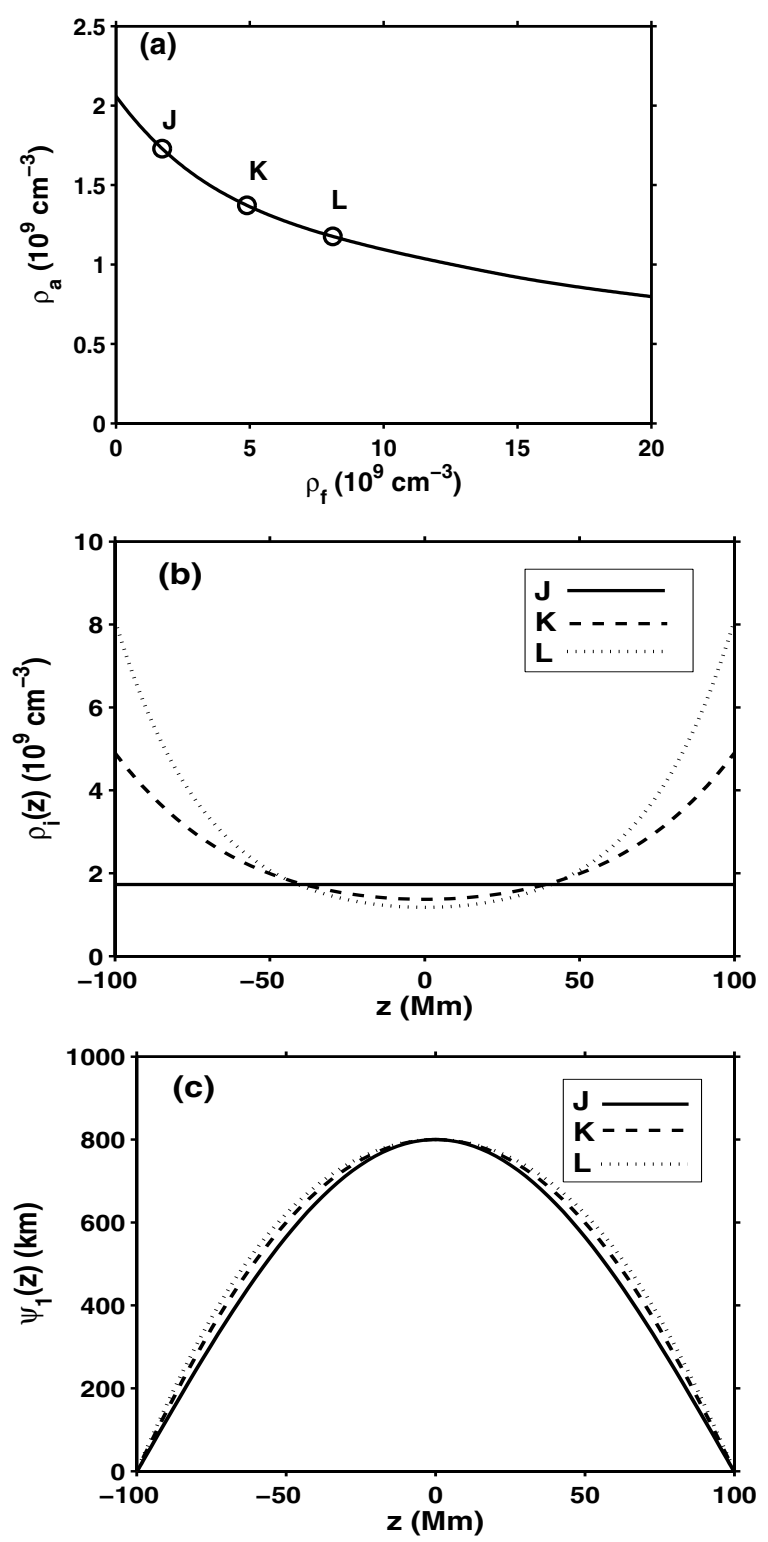

Fig. 7. a) For the exponentially stratified density profile (13), the allowed density pairs for the fundamental standing mode are shown where $\rho_{\mathrm{a}}$ and $\rho_{\mathrm{f}}$ are apex and footpoint densities. The fixed parameters are $L=100 \mathrm{Mm}, B=10 \mathrm{G}, P=5$ min and $\epsilon=0.1$. Pairs J, K and $\mathrm{L}$ are chosen to represent $H=\infty, H=50 \mathrm{Mm}$ and $H=33 \mathrm{Mm}$. b) The density profiles of $\mathrm{J}, \mathrm{K}$ and $\mathrm{L}$. c) The amplitude profiles of $\mathrm{J}, \mathrm{K}$ and $\mathrm{L}$, where for all, the apex maximum amplitude for the fundamental kink mode (at $z=0$ ) is normalised to $A_{1}=800 \mathrm{~km}$.

the longitudinal direction. A loop footpoint segment could be brightly visible in one specific temperature passband but if the loop becomes hotter towards the loop apex it will appear fainter or may even disappear out of the passband altogether. For this model to be really useful we would ideally like the whole loop to be visible while it is oscillating. The planned Atmospheric Imaging Assembly (AIA) EUV imager on the Solar Dynamics Observatory (SDO) mission may be helpful in this regard as it will view with seven different EUV passbands simultaneously, but unfortunately the resolution is only equivalent to that of TRACE.

Acknowledgements. The authors thank Prof. Ruderman for a number of useful discussions. R.E. acknowledges M. Kéray for patient encouragement. The authors are also grateful to NSF, Hungary (OTKA, Ref. No. TO43741) and the Engineering and Physical Sciences Research Council (EPSRC), UK for the financial support they received.

\section{References}

Abramowitz, M., \& Stegun, I. A. 1965, Handbook of Mathematical Functions (New York: Dover Publications)

Andries, J., Arregui, I., \& Goossens, M. 2005a, ApJ, 624, L57

Andries, J., Goossens, M., Hollweg, J. V., Arregui, I., \& Van Doorsselaere, T. 2005b, A\&A, 430, 1109

Aschwanden, M. J. 2004, Physics of the Solar Corona: An Introduction (Berlin: Springer-Verlag)

Aschwanden, M. J., Fletcher, L., Schrijver, C. J., \& Alexander, D. 1999a, ApJ, 520,880

Aschwanden, M. J., Newmark, J. S., Delaboudinière, J., et al. 1999b, ApJ, 515, 842

Aschwanden, M. J., De Pontieu, B., Schrijver, C. J., \& Title, A. M. 2002, Sol. Phys., 206, 99

Bennett, K., Roberts, B., \& Narain, U. 1998, Sol. Phys., 185, 41

Cargill, P. J., \& Klimchuk, J. A. 1997, ApJ, 478, 799

Coddington, E. A., \& Levinson, N. 1955, Theory of Ordinary Differential Equations (New York: McGraw-Hill)

Cowling, T. G. 1960, Magnetohydrodynamics (New York: Interscience)

Díaz, A. J., Oliver, R., \& Ballester, J. L. 2002, ApJ, 580, 550

Dymova, M. V., \& Ruderman, M. S. 2005, Sol. Phys., 229, 79

Edwin, P. M., \& Roberts, B. 1983, Sol. Phys., 88, 179

Erdélyi, R., \& Carter, B. 2006, A\&A, 455, 361

Erdélyi, R., \& Fedun, V. 2006, Sol. Phys., 238, 41

Golub, L., \& Pasachoff, J. M. 1997, The Solar Corona (Cambridge: Cambridge University Press)

Jain, R., \& Yashiro, S. 2002, A\&A, 394, 1111

Klimchuk, J. A., Antiochos, S. K., \& Norton, D. 2000, ApJ, 542, 504

Landau, L. D., \& Lifshitz, E. F. 1956, Electrodynamics of Continuous Media (New York: Pergamon Press)

Nakariakov, V. M., Ofman, L., DeLuca, E. E., Roberts, B., \& Davila, J. M. 1999, Science, 285,862

Reale, F., \& Peres, G. 2000, ApJ, 528, L45

Roberts, B., Edwin, P. M., \& Benz, A. O. 1984, ApJ, 279, 857

Ruderman, M. S. 2003, A\&A, 409, 287

Schmelz, J. T., Nasraoui, K., Richardson, V. L., et al. 2005, 627, L81

Van Doorsselaere, T., Debosscher, A., \& Poedts, S. 2004, A\&A, 424, 1065

Vernazza, J. E., Avrett, E. H., \& Loeser, R. 1981, ApJS, 45, 635

Verwichte, E., Nakariakov, V. M., Ofman, L., \& Deluca, E. E. 2004, Sol. Phys., 223,77

Watko, J. A., \& Klimchuk, J. A. 2000, Sol. Phys., 193, 77

Zwillinger, D. 1997, Handbook of Differential Equations (Orlando: Academic Press) 